\title{
Clear-cell renal cell carcinoma single thyroid metastasis: A single-center retrospective analysis and review of the literature
}

\author{
Isabella Ricci ${ }^{1}$, Francesco Barillaro ${ }^{2}$, Enrico Conti ${ }^{2}$, Donatella Intersimone ${ }^{3}$, Paolo Dessanti ${ }^{3}$, \\ Carlo Aschele ${ }^{1}$ \\ ${ }^{1}$ Department of Oncology, Ospedale S. Andrea, La Spezia, Italy; \\ ${ }^{2}$ Department of Urology, Ospedale S. Bartolomeo, Sarzana, Italy; \\ ${ }^{3}$ Department of Pathology, Ospedale S. Andrea, La Spezia, Italy.
}

\begin{abstract}
Summary Renal cell carcinoma (RCC) is known to cause metastasis to unusual sites, which can be both synchronous or metachronous. Thyroid gland is a rare site for metastasis. However, RCC is the most common primary neoplasm to metastasize to the Thyroid gland. Report of three cases and review of the literature.
\end{abstract}

KEY WORDS: Renal cell carcinoma; Thyroid; Metastasis.

Submitted 26 June 2020; Accepted 20 August 2020

\section{INTRODUCTION}

Kidney cancer accounts for 5\% and 3\% of all adult malignancies in men and women, respectively, representing the $7^{\text {th }}$ most common cancer in men and the $10^{\text {th }}$ most common cancer in women. Renal cell carcinoma (RCC) accounts for $80 \%$ of all kidney cancers. Common metastatic sites of RCC are lung, lymph nodes, bone and liver. RCC is responsible of unusual metastatic sites, although it is the most common primary neoplasm that metastasizes to thyroid gland (1). The incidence of thyroid metastasis has been reported to be higher on autopsy studies (2) and ranges from $0.5 \%$ to $24 \%$ in high stage malignancies. Metastasis can occur many years after initial diagnosis, but are extremely rare in clinical practice. A thyroid nodule in a patient with a history of RCC should be considered potentially metastatic. We retrospectively reviewed our database searching patients who developed recurrence of RCC with thyroid metastases. The total number of RCC patients observed from 2004 to 2019 in our institution was of about 208 cases. Out of these, only three cases developed recurrence of RCC with thyroid metastases many years after nephrectomy in a 15 year follow-up range (3).

\section{Case report/Case presentation}

\section{Case 1}

A 61-year-old female admitted to our hospital in April 2004, presented with left flank pain and history of weight loss. An MRI was performed showing an exophytic, hypervascular, solid mass, measuring $7 \times 8 \mathrm{~cm}$. The MRI findings were in keeping with a malignant renal tumor.
Two weeks later, the patient underwent left radical nephrectomy. The histopathological examination reported clear-cell renal cell carcinoma. TNM staging was T3a with G3 Fuhrman grade. She was followed up by the oncologist for 10 years without evidence of recurrence. After ten years, a PET/CT was performed demonstrating a metabolically active area in the thyroid right lobe. Ultrasound imaging confirmed the presence of a solid hypoechoic, well-demarcated nodule of $2.2 \mathrm{~cm}$. Decision was made to perform a radical thyroidectomy in November 2014.

The histopathological examination showed metastatic RCC of clear cell type (Figure 1A). Three year after thyroidectomy, in a further follow-up, two pulmonary nodules were detected and consequently the patient underwent thoracotomy for atypical double resection of the upper lobe and left pulmonary lingula. TNM staging was Tla and histology showed lung lipid adenocarcinoma (Figure 1E, F) while the other nodule instead was comparable with metastasis from RCC (Figure 1G, H).

The subsequent follow-up was negative. The average latency time before the detection of thyroid metastases was 10 years (4).

\section{Case 2}

A76-year-old male underwent a right radical nephrectomy for a solid mass clear cell renal carcinoma in March 2016. TNM staging was T3aNOMO. In April 2018 he underwent laparoscopy and subsequently to lower pole resection of the left kidney. The histopathological examination reported papillary carcinoma. TNM staging Tlb G2. One year after surgery, a solid nodule in the right lobe of the thyroid gland $(4.5 \times 3 \times 3.5 \mathrm{~cm})$ was detected. The patient presented a painful mass associated with cough and dysphagia. Size of metastases was significantly higher in this patient compared to those with painless mass. Ultrasound imaging followed by computed tomography (CT) showed a well-defined hypodense nodule in the right lobe of the thyroid gland. Our 79-year-old patient underwent thyroidectomy, after FNA cytology, in 2019. The histopathological examination reported metastatic RCC of clear cell type which developed in his third follow-up year. The subsequent follow-up was negative. The average latency time before the detection of thyroid metastases was 3 years.

No conflict of interest declared. 


\section{Figure 1.}

A: Thyroid (bottom left) with metastasis of adult renal cell carcinoma clear cell (conventional) (top right).

Hematoxylin Eosin 40x.

B: Thyroid (upper half) with nodular hyperplasia and with metastasis of adult renal cell carcinoma (lower half). Hematoxylin Eosin 20x.

$C-D$ : Renal cell metastasis showed immunoreactivity for RCC/Renal cell carcinoma marker (c) and negativity for thyroglobulin (d), which was positive in the thyroid parenchyma. E-F: Lung metastasis (lingular lobe left lung) of renal cell carcinoma. Hematoxylin Eosin 20x (e). Lung metastasis (lingular lobe left lung) of renal cell carcinoma.

Hematoxylin Eosin 20x (f).

G-H: Lung adenocarcinoma (lower lobe left lung) with predominantly lepidic type growth. Napsin A 40x (g). The neoplastic cells show intense nuclear immunoreactivity for Pax 8 confirming their renal origin. Pax 8, 20x (h).

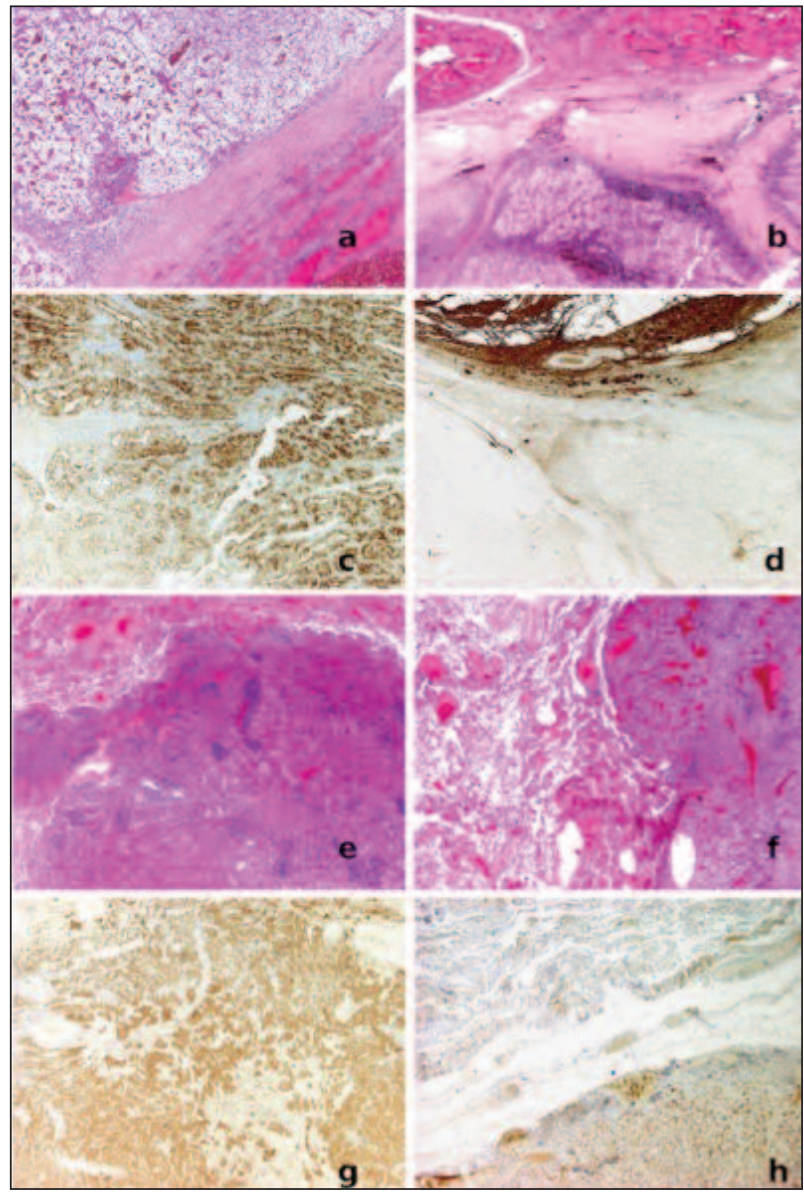

Case 3

A 78 year-old female presented with a history of a lump on the neck for the last 3 months in May 2014. The past medical history encompassed RCC on the right side around 10 years before. At that time she has been treated surgically undergoing left radical nephrectomy and left adrenalectomy for a large renal mass measuring $11 \times 7 \times 7 \mathrm{~cm}$. The histology showed clear cell RCC G2 sec Fuhrman. Tumor was not infiltrating through the capsule. Surgical margins were free of tumor and TNM staging T2bNOMO. She was followed-up by the oncologist for almost 10 years and no evidence of recurrence was noted. The examination of the neck revealed evidence of a $3 \mathrm{~cm}$ nodule in the right thy- roid lobe. On positron emission tomography/computed tomography there was a metabolically active area in the thyroid right lobe. Ultrasound imaging confirmed a solide hypoechoic nodule. The patient subsequently underwent fine needle aspiration cytology which showed borderline neoplastic cells, although it poses the diagnostic doubt. A thyroidectomy was therefore carried out. The istopathological examination showed metastatic RCC of clear cell type (Figure 1B). Mean latency time before the detection of thyroid metastases was 10 years. The subsequent follow-up was negative.

\section{Discussion}

RCC accounts for approximately 3-4\% of all adult malignancies. It is more common in males and occurs predominantly in the $6^{\text {th }}$ to $8^{\text {th }}$ decade of life.

Major histopathological subtypes include clear cell carcinoma, papillary carcinoma, chromophobe carcinoma, medullary carcinoma. The metastasis may be detected at the time of diagnosis (synchronous) or may be found years after the diagnosis and treatment (metachronous) (5-11). It has been estimated that $20-30 \%$ of patients including those who have undergone nephrectomy with curative intent will develop recurrence and out of these 50\% will relapse distantly (6). Moreover, solitary metastasis from RCC occurs with an incidence rate of about $1-4 \%$ of which about $1 \%$ occur in the thyroid gland. Most of the recurrences appears within 3 years from surgery, but delayed recurrences even after decades have been reported (7). Characteristics of patients described in literature are reported in Table 1 (11). RCC metastasis to thyroid generally are symptomatic or painless mass but can be also completely asymptomatic and discovered incidentally during follow-up. Size of metastases was higher in symptomatic patients compared to those with painless mass and asymptomatic ones. Rarely may present dysphagia, dysphonia or dyspnea. Moreover, there was a weak correlation between lag time and size of metastases.

Table 1.

Characteristics of patients described in literature.

\begin{tabular}{|l|c|}
\hline \multicolumn{2}{|l|}{ Characteristics of patients } \\
\hline Agge (yr) mean \pm SD & $64 \pm 10$ \\
Male & $64 \pm 11$ \\
Female & $47 \%(69)$ \\
\hline Sex percentage (number of patients) & $53 \%(77)$ \\
Male & $32 \%(21)$ \\
Female & $29 \%(19)$ \\
\hline Initial CCRCC stage & $31 \%(20)$ \\
Stage I CCRCC & $8 \%(5)$ \\
Stagee II CCRCC & \\
Stage III CCRCC & \\
Stage IV CCRCC & \\
\hline Lag time (years) from initial diagnosis of CCRCC & \\
$8.7 \pm 6.5$ mean \pm SD & $30 \%(33)$ \\
\hline Symptoms & $70 \%(76)$ \\
Asymptomatic & $62 \%(75)$ \\
Symptomatic or painless mass & $38 \%(46)$ \\
\hline Surgery & \\
Total thyroidectomy & \\
Subtotal thyyroidectomy & \\
\hline
\end{tabular}


Regarding the tests performed for the diagnosis of RCC metastases to the thyroid gland, ultrasound of the neck was the most frequently used imaging tecnique followed by computed tomography (CT scan) and positron emission tomography (PET scan). Usually metastatic thyroid lesions appear as solid hypoechoic, well-demarcated nodules with irregular vascularity on ultrasound imaging and cold nodules on radioisotope uptake studies. These radiological features are not specific and it is not possible to distinguish between primary and secondary thyroid neoplasms on imaging. Only a case demonstrated incidental thyroid abnormalities on positron emission tomography/computed tomography and ultrasound later confirmed as a metastases of renal cell carcinoma. Fine needle aspiration (FNA) cytology is necessary to establish preoperative diagnosis. A challenge it is sometimes distinguishing metastasis from tumors of thyroid, because can have clear cell component on FNA cytology alone. In these cases, immunohistochemistry is helpful and aids in differential diagnosis.

In our cases cytocheratin, vimentin and CD 10, traditional immunohistochemical markers for renal cell carcinoma, were positive; thyroglobulin, thyroid transcription factor-1 (TTF-1), and calcitonin, markers used for identifying primary thyroid malignancies, were negative (Figure 1C, D). Definitive diagnosis of metastatic RCC is usually made by histopathological examination after thyroidectomy (8). Surgical resection with either partial or radical thyroidectomy should be performed if thyroid gland is the only site for metastasis. Prognosis is good in this group $(9,10)$. Thyroid metastasis from RCC has a better survival rate according to the Literature. Patients with disseminated disease have poor prognosis and should undergo thyroidectomy only for palliation for compressive symptoms. Treatment choice in metastatic renal cell carcinoma depends on different factors including the extent of the disease and prognostic risk factors such as Karnofsky performance status, diagnosis timeline and laboratory findings. Direct treatments to the thyroid metastasis result in prolonged survival especially in solitary thyroid gland metastasis where surgical treatment is recommended. It is demonstrated a favorable prognosis in patients treated with radical surgery (average 5-year survival rate 30-60\%). Clinically it has been observed that the overall survival rate in patients undergoing thyroidectomy for metastases of RCC is more affected by general health status rather than by tumor-related factors.

Sunitinib could be effective for the treatment of these metastases. His mechanism of action is based on causing reduction in thyroid volume. Negative adverse reaction of this drug can results in a various grade of thyroid dysfunction from hypothyroidism to thyroid atrophy, in particular in patients who receive the drug over a long period of time, and that could result in irreversible hypothyroidism. This is thought to be a result of a possible sunitinib-induced thyrotoxicosis along with a direct effect of the drug which could cause degeneration of thyroid follicular cells.

To understand the possible effectiveness of Sunitinib on thyroid further investigations are needed.

\section{Conclusions}

A thyroid nodule in a patient with a history of RCC should be considered as potentially metastatic. It's chal- lenging to distinguish between primary and secondary thyroid neoplasms on imaging, and clinical manifestation and radiographic findings are non-specific. FNA cytology and immunohistochemistry are helpful in establishing diagnosis and should be obtained in suspected cases. The average latency time before the detection of thyroid metastases was variable. However, a lifelong follow-up is recommended. Our cases demonstrate the importance of considering RCC metastases to the thyroid even years after nephrectomy to avoid potential delays in diagnosis (11).

\section{REFERENCES}

1. Chen H, Nicol TL, Udelsman R. Clinically significant, isolated metastatic disease to the thyroid gland. World J Surg. 1999; 23:177-80.

2. Willis RA. Metastatic tumours in the thyroid gland. Am J Pathol. 1931; 7:187-208.3.

3. Bayrakter Z, Albayrak S. Metastasis of renal cell carcinoma to the thyroid gland 9 years after nephrectomy: a case report and literature review. Arch Ital Urol Androl. 2017; 89:151-153.

4. Cilengir AH, Kalayci TO, Duygulu G, et al. Metastasis of renal clear cell carcinoma to thyroid gland mimicking adenomatous goiter. Pol J Radiol. 2016, 81:618-621.

5. Motzer RJ, Bander NH, Nanus DM. Renal cell carcinoma. NEJM. 1996. 335:865-875.

6. Flanigan RC, Campbell SC, Clark JI, et al. Metastatic renal cell carcinoma Curr Treat Options Oncol. 2003; 4:385-390.

7. Eggener SE, Yossepowitch O, Pettus JA, et al. Renal cell carcinoma recurrence after nephrectomy for localized disease: predicting survival from time of recurrence, JCO. 2006; 24:3101-3106.

8. Aljiabri KS, Bokhari SA, Fadag RB, et al. Thyroid metastasis from renal cell carcinoma, Archives of endocrinology and diabetes care. 2018; 1:65-70.

9. Chung AY, Tran TB, Brumund KT, et al. Metastases to the tyroid: a review of the literature from the last decade. Thyroid. 2012; 22:258-68

10. De Stefano R, Carluccio R, Zanni E, et al, Management of thyroid nodules as secondary involvement of renal cell carcinoma case report and literature review, Anticancer research. 2009; 29:473-6.

11. Khaddour K, Marernych N, Ward WL, et al. Characteristics of clear cell renal cell carcinoma metastases to the thyroid gland: a systematic review, World J Clin Cases. 2019; 7:3474-3485.

\section{Correspondence}

Isabella Ricci, MD (Corresponding Author)

isabella.ricci@as15.liguria.it

Carlo Aschele, MD

carlo.aschele@asl5.liguria.it

Department of Oncology, Ospedale S. Andrea, La Spezia (Italy)

Francesco Barillaro, MD

francesco.barillaro@asl5.liguria.it

Enrico Conti, MD

enrico.conti@as15.liguria.it

Department of Urology, Ospedale S. Bartolomeo, Sarzana (Italy)

Donatella Intersimone, MD

donatella.intersimone@as15.liguria.it

Paolo Dessanti, MD

paolo.dessanti@asl5.liguria.it

Department of Pathology, Ospedale S. Andrea, La Spezia (Italy) 